\title{
THE INDEX THEOREM OF LATTICE WILSON-DIRAC OPERATORS VIA HIGHER INDEX THEORY
}

\author{
YOSUKE KUBOTA \\ Department of Mathematical Sciences, Shinshu University \\ 3-1-1 Asahi, Matsumoto, Nagano, 390-8621, Japan \\ and \\ iTHEMS Program, RIKEN \\ 2-1 Hirosawa, Wako, Saitama, 351-0198, Japan
}

\begin{abstract}
Aвstract. We give a proof of the index theorem of lattice Wilson-Dirac operators, which states that the index of a twisted Dirac operator on the standard torus is described in terms of the corresponding lattice Wilson-Dirac operator. Our proof is based on the higher index theory of almost flat vector bundles.
\end{abstract}

\section{Contents}

1. Introduction

2. Lattice covariant derivative and group quasi-representation

3. K-theory of the universal hermitian Wilson-Dirac operator

4. Wilson-Dirac index theorem as almost flat index pairing

5. Generalizations

References

\section{InTRODUCTION}

Lattice gauge theory is a theoretical and numerical approach for studying the Yang-Mills gauge theory, especially the quantum chromodynamics, in which the (Wick-rotated Euclidean) spacetime is approximated by its discrete lattice. If we also assume that the spacetime is compact by imposing the periodic boundary condition, the lattice becomes a finite number of points. This approximation makes the path-integral finite-dimensional and hence calculable by e.g. the Monte Carlo simulation.

The main subject of this paper is the lattice approximation of the chiral anomaly in gauge theory. The chiral anomaly is the quantum breaking of the gauge symmetry, which the classical Yang-Mills theory possesses. According to Fujikawa's method, it is described by the Fredholm index of the Dirac operator twisted by a vector

E-mail address: ykubota@shinshu-u.ac.jp.

Date: September 8, 2020.

2010 Mathematics Subject Classification. Primary 19K56; Secondary 81T13, 46L80.

Key words and phrases. Wilson-Dirac operator, lattice gauge anomaly, operator K-theory, group quasi-representation. 
bundle. Here a natural question arises: what is a lattice approximation of the chiral anomaly, or equivalently, the index of the twisted Dirac operator? A main difficulty for answering this question is that, although the Fredholm theory is essentially an infinite dimensional phenomenon, the approximated lattice Dirac operator acts on the finite dimensional Hilbert space of $\ell^{2}$-functions on the lattice.

The answer is given in the literature of theoretical physics [NN95, HLN98] by considering the lattice (hermitian) Wilson-Dirac operator, a variation of the lattice Dirac operator defined by adding a new term called the Wilson term. If the lattice scale is sufficiently fine, we obtain an invertible self-adjoint matrix by imposing a mass term to the hermitian Wilson-Dirac operator (this estimate is studied by Neuberger [Neu00]). Then the half difference of the number of its positive and negative eigenvalues, instead of the number of the zero-modes, is a topological invariant.

This integer actually coincides with the index of the Dirac operator at the continuum limit. This equality has been studied in the literature of theoretical physics [Lüs99,Suz99, Fuj99, Ada02a]. In this paper we shed new light on this index theorem from the viewpoint of higher index theory of almost flat vector bundles, i.e., a vector bundle equipped with a hermitian connection whose curvature is small. We mention that there are several other mathematical approaches to the same problem [Yam20, $\left.\mathrm{FFM}^{+}\right]$.

Another motivation of the paper is the study of almost commuting unitary matrices. Motivated from a question by Halmos [Hal76], Voiculescu [Voi83] constructs an example of a pair of unitary matrices $(U, V)$ such that the norm of the commutator $\|[U, V]\|$ is arbitrarily small but not perturbed to any commutative pair. Excel-Loring [EL91] gives an alternative proof using a topological invariant motivated from $\mathrm{C}^{*}$-algebra $\mathrm{K}$-theory. As a by-product of the proof of the lattice index theorem, we give an explicit formula of this invariant inspired from the hermitian Wilson-Dirac operator (Theorem 3.14).

This topological invariant is related to the geometry of almost flat vector bundles by Connes-Gromov-Moscovici [CGM90]. A $d$-tuple of mutually almost commuting unitaries is thought of as a quasi-representation of the group $\mathbb{Z}^{d}$. Generalizing the monodromy correspondence of flat bundles and representations, almost flat vector bundles on a space $M$ corresponds roughly in one-to-one with a quasirepresentation of $\pi_{1}(M)$. It is clarified in the work of Hanke-Schick [HS06] and Dadarlat [Dad12] that the K-theoretic invariant of a group quasi-representation coincides with the index of Dirac operators twisted by the corresponding almost flat bundles. This fact plays a key role in our proof of the index theorem of Wilson-Dirac operators.

1.1. Statement of the theorem. Let $d$ be a positive integer, standing for the dimension of the spacetime. For simplicity of discussion, here we only consider the case that $d$ is even (for a more general case, see Subsection 5). Let $V$ be the $d$-dimensional Euclidean space. That is, $V$ is the linear space $\mathbb{R}^{n}$ equipped with the inner product $\langle\cdot, \cdot\rangle$. Let $\Pi$ be the standard lattice of $V$, i.e., a cocompact rank $d$ free abelian subgroup $\Pi \subset V$ generated by an orthonormal basis $t_{1}, \cdots, t_{n}$ of $V$. Set $M:=V / \Pi$. It is equipped with the induced Riemannian metric, denoted by $g$.

Let $\mathbb{C} \ell(-V)$ denote the Clifford algebra of $V$ with the negative definite inner product. That is, $\mathbb{C} \ell(-V)$ is the universal $\mathbb{C}$-algebra generated by $\{c(v) \mid v \in V\}$ with the relations $c(v) c(w)+c(w) c(v)=-2\langle v, w\rangle 1$. It is equipped with the 
$\mathrm{C}^{*}$-algebra structure by the adjoint $c\left(v^{*}\right)=-c(v)$. Since $d$ is even, the algebra $\mathbb{C} \ell(-V)$ has a unique irreducible $*$-representation $S$.

Let $E$ be a complex vector bundle on $M$ equipped with a hermitian inner product and let $\nabla$ be a hermitian connection on $E$. Then the twisted Dirac operator $D^{E}$ is defined as

$$
D^{E}=\sum_{i=1}^{n} c\left(v_{i}\right) \nabla_{v_{i}}: \Gamma(M, E \otimes S) \rightarrow \Gamma(M, E \otimes S),
$$

where $v_{1}, \cdots, v_{n}$ is a standard basis of $T_{0} V \cong V$ corresponding to $t_{1}, \cdots, t_{n}$. Then $D^{E}$ is an odd self-adjoint operator with respect to the $\mathbb{Z}_{2}$-grading of $S$. Let $D_{0}^{E}$ and $D_{1}^{E}$ denote the off-diagonal entries of $D^{E}$ with respect to the $\mathbb{Z}_{2}$-grading $\gamma$ as $D_{E}=\left(\begin{array}{cc}0 & D_{1}^{E} \\ D_{0}^{E} & 0\end{array}\right)$. Our main concern, the Fredholm index of $D^{E}$, is the integer

$$
\operatorname{Index}\left(D^{E}\right):=\operatorname{dim} \operatorname{ker}\left(D_{0}^{E}\right)-\operatorname{dim} \operatorname{ker}\left(D_{1}^{E}\right),
$$

which is well-defined since $D^{E}$ is an elliptic operator over a compact manifold $M$.

Now we introduce a lattice approximation of the operator $D^{E}$. Firstly, we replace the covariant derivative $\nabla_{v_{j}}$ in (1.1) with a difference operator. For a path $\gamma:[0, t] \rightarrow M$, we write $\Gamma_{\gamma}^{E}: E_{\gamma(0)} \rightarrow E_{\gamma(1)}$ for the parallel transport of $(E, \nabla)$ along $\gamma$. Let $a>0$ stand for the scale of the lattice approximation (hence its is an inverse integer; $a=1 / N)$. Then the approximated spacetime is the quotient $a \Pi / \Pi \subset M$ of the finer lattice $a \Pi$. Now the shift operator in $j$-th direction is defined by the direct sum

$$
U_{j}^{a, E}:=\bigoplus_{x \in a \Pi / \Pi}\left(\Gamma_{\left[x, x+a v_{j}\right]}^{E}: E_{x} \rightarrow E_{x+a v_{j}}\right),
$$

which is a unitary operator on $\bigoplus_{x \in a \Pi / \Pi} E_{x}$.

Definition 1.3. The lattice covariant derivative $\nabla^{a, E}$, the lattice Dirac operator $D^{a, E}$, the lattice Wilson term $W^{a, E}$ and the lattice hermitian Wilson-Dirac operator $D_{W}^{a, E}$ with the scale $a=1 / N$ are defined as following;

$$
\begin{aligned}
\nabla_{j}^{a, E} & :=\left(U_{j}^{a, E}-1\right) / a, \\
D^{a, E} & :=\sum_{j} c\left(v_{j}\right)\left(\nabla_{j}^{a, E}-\left(\nabla_{j}^{a, E}\right)^{*}\right) / 2, \\
W^{a, E} & :=\sum_{j}\left(\nabla_{j}^{a, E}+\left(\nabla_{j}^{a, E}\right)^{*}\right) / 2, \\
D_{W}^{a, E} & :=\gamma W^{a, E}+D^{a, E} .
\end{aligned}
$$

Note that $D^{a, E}, W^{a, E}$ and $D_{W}^{a, E}$ acts on the Hilbert space

$$
\mathcal{H}^{a, E}:=\left(\bigoplus_{x \in a \Pi / \Pi} E_{x}\right) \otimes S .
$$

Morover, we impose the mass term $m_{0} \gamma$ to obtain the massive Wilson-Dirac operator $D_{W}^{a, E}+m_{0} \gamma$. In this paper we consider the following two kinds of the mass term.

(1) The cut-off scale mass $m_{0}=m / a$.

(2) The constant mass term $m$ independent of the length-scale $a$. 
The massive hermitian Wilson-Dirac operator considered in the context of theoretical physics is mainly (1), or its functional calculus $\left(D_{W}^{a, E}+\frac{m}{a}\right) /\left|D_{W}^{a, E}+\frac{m}{a}\right|$ (this operator is called the overlap Dirac operator). On the other hand, the operator (2) is also considered in recent researches $\left[\mathrm{FKM}^{+} 19, \mathrm{FKM}^{+} 20\right]$. We show the index theorem for both of these operators.

We define the "index" of the massive hermitian Wilson-Dirac operator as following.

Definition 1.4. For a self-adjoint invertible matrix $A$, we define $\mathbf{I}(A)$ as

$$
\mathbf{I}(A):=\frac{\operatorname{dim} E_{>0}(A)-\operatorname{dim} E_{<0}(A)}{2},
$$

where $E_{>0}(A)$ (resp. $E_{<0}(A)$ ) denotes the spectral subspaces of $A$ corresponding to positive (resp. negative) eigenvalues.

Remark 1.5. Assume that the (finite rank) Hilbert space on which $A$ acts is equipped with a $\mathbb{Z}_{2}$-grading $\gamma$ such that the even and odd subspaces are isomorphic (the space $\mathcal{H}^{a, E}$ above satisfies this assumption). Then we have

$$
\mathbf{I}(A)=\operatorname{dim} E_{>0}(A)-\operatorname{dim} E_{>0}(\gamma) .
$$

This holds because $\operatorname{dim} E_{>0}(A)+\operatorname{dim} E_{<0}(A)=\operatorname{dim} \mathcal{H}^{a, E}$ and $\operatorname{dim} E_{>0}(\gamma)=$ $\operatorname{dim} \mathcal{H}^{a, E} / 2$.

Theorem 1.6. The following hold.

(1) For any $0<m<2$, there is a length scale $a_{0}=1 / N_{0}$ such that $D_{W}^{a, E}+\frac{m}{a}$ is invertible and

$$
\mathbf{I}\left(D_{W}^{a, E}+\frac{m}{a} \gamma\right)=\operatorname{Index}\left(D^{E}\right)
$$

holds for any $0<a<a_{0}$.

(2) There is $m_{0}>0$ such that, for any $m>m_{0}$ there is a length scale $a_{0}=1 / N_{0}$ such that the operator $D_{W}^{a, E}+m \gamma$ is invertible and

$$
\mathbf{I}\left(D_{W}^{a, E}+m \gamma\right)=\operatorname{Index}\left(D^{E}\right)
$$

holds for any $0<a<a_{0}$.

The strategy of the proof is as follows. Firstly, in Section 2, we relate the lattice shift operator (1.2) with the quasi-representation $\pi_{a, E}$ of $\Pi=\pi_{1}(M)$ obtained by the constructions due to Gromov-Lawson [GL83] and Connes-GromovMoscovici [CGM90]. This enables us to understand the Wilson-Dirac operator $D_{W}^{a, E}$ as the image of the "universal" element $\hat{D}_{W}$ of the matrix-coefficient group algebra $\mathbb{C}[\Pi] \otimes \operatorname{End}(S)$ through $\pi_{a, E}$, as is done in Section 3. In particular, here we show that $\hat{D}_{W}^{a, E}+m \gamma$ is an invertible self-adjoint operator representing the Bott element $\beta \in \mathrm{K}_{0}\left(C^{*} \Pi\right) \cong \mathrm{K}^{0}(\hat{\Pi})$. Finally, in Section 4 , we relate $\pi_{a, E}(\beta)$ with the index of $D^{E}$ by applying the argument of Hanke-Schick [HS06].

After proving Theorem 1.6, in Section 5, we discuss two generalizations of this theorem. The first is the lattice family index, which has been considered by Adams [Ada02b]. The second is the lattice Real and Clifford equivariant index. This generalization is also studied in the forthcoming paper by Fukaya et. al. [ $\left.\mathrm{FFM}^{+}\right]$. 
Acknowledgement. The author would like to thank Mikio Furuta for his inspiring seminar talk on the Wilson-Dirac operator at RIKEN. This work was supported by RIKEN iTHEMS and JSPS KAKENHI Grant Numbers 19K14544, JPMJCR19T2, $17 \mathrm{H} 06461$.

\section{LATTICE COVARIANT DERIVATIVE AND GROUP QUASI-REPRESENTATION}

In this section we relate the lattice covariant derivative $\nabla_{j}^{a, E}$ given in Definition 1.3 with the quasi-representation of the group $\Pi$ obtained by a combination of the Gromov-Lawson construction and the almost monodromy correspondence.

2.1. Group quasi-representation and almost monodromy. We start with a brief review of group quasi-representations, almost flat bundles and their almost monodromy correspondence. For a more detail on this subsection, we refer the reader to [CGM90, HS06, CD13, Dad14, Kub20].

In general, for a finitely presented discrete group $\Gamma$ and its finite set of generators $\mathcal{G}$, we say that a map $\pi: \Gamma \rightarrow \mathrm{U}(P)$ is a $(\varepsilon, \mathcal{G})$-representation of $\Gamma$ on $P$ if $\pi(e)=1$ and

$$
\|\pi(g) \pi(h)-\pi(g h)\|<\varepsilon
$$

for any $g, h \in \mathcal{G}$ such that $g h \in \mathcal{G}$. Our interest is quasi-representations of the group $\Pi \cong \mathbb{Z}^{d}$ with respect to the generating set $\mathcal{G}=\left\{e, t_{i}^{ \pm 1}, t_{i}^{ \pm 1} t_{j}^{ \pm 1} \mid i, j=\right.$ $1, \cdots, n\}$. Then an $(\varepsilon, \mathcal{G})$-representation $(\pi, \mathcal{H})$ of $\Pi$ corresponds to a family of unitary operators $U_{j}:=\pi\left(t_{j}\right)$ which are mutually almost commutative, namely $\left\|\left[U_{j}, U_{l}\right]\right\|<2 \varepsilon$. Conversely, if we have a mutually $\varepsilon$-commutative $d$-tuple of unitaries, then $\pi\left(t_{j}^{ \pm 1}\right)=U_{j}^{ \pm 1}$ and $\pi\left(t_{j}^{ \pm 1} t_{l}^{ \pm 1}\right):=U_{j}^{ \pm 1} U_{l}^{ \pm 1}$ for $j<l$ determines a $(2 \varepsilon, \mathcal{G})$-representation of $\Pi$. A typical example of almost commuting unitaries in $d=2$ is

$$
U_{1}=\left(\begin{array}{ccccc}
1 & 0 & 0 & \cdots & 0 \\
0 & \zeta_{n} & 0 & \cdots & 0 \\
0 & 0 & \zeta_{n}^{2} & \cdots & 0 \\
\vdots & \vdots & \vdots & \ddots & \vdots \\
0 & 0 & 0 & \cdots & \zeta_{n}^{n-1}
\end{array}\right), U_{2}=\left(\begin{array}{ccccc}
0 & 0 & \cdots & 0 & 1 \\
1 & 0 & \cdots & 0 & 0 \\
0 & 1 & \cdots & \vdots & 0 \\
\vdots & \vdots & \ddots & 0 & \vdots \\
0 & \cdots & 0 & 1 & 0
\end{array}\right),
$$

where $\zeta_{n}$ denotes the root of unity $e^{2 \pi i / n}$.

There is a fruitful construction, established by Connes-Gromov-Moscovici in [CGM90], of a quasi-representation of the fundamental group $\pi_{1}(M)$ of a manifold $M$ from an almost flat bundle on $M$ as the monodromy "representation". A pair $(E, \nabla)$ is said to be a $(\varepsilon, g)$-flat vector bundle on $M$ if $E$ is a hermitian vector bundle on $M$ and $\nabla$ is a hermitian connection on $E$ whose curvature tensor $R^{E} \in \Omega^{2}(M$, End $E)$ satisfies

$$
\left\|R_{E}\right\|:=\sup _{x \in M} \sup _{\xi \in \bigwedge^{2} T_{x} M \backslash\{0\}} \frac{\left\|R_{E}(\xi)\right\| \operatorname{End}\left(E_{x}\right)}{\|\xi\|}<\varepsilon .
$$

As in Section 1, we denote by $\Gamma_{\gamma}^{\nabla}$ the parallel transport on $E$ along a path $\gamma:[0,1] \rightarrow M$ with respect to the connection $\nabla$. Let us choose a collection of closed loops $\ell_{j} \in \Omega M$ such that the set $\mathcal{G}:=\left\{\left[\ell_{j}\right]\right\}$ generates $\pi_{1}(M)$. 
Definition 2.1. The collection of operators

$$
\pi_{E}\left(\left[\ell_{j}\right]\right):=\Gamma_{\ell_{j}}^{\nabla}: E_{0} \rightarrow E_{0}
$$

forms a $(C \varepsilon, \mathcal{G})$-representation for some $C>0$ depending only on a choice of $\left\{\ell_{j}\right\}$ and $g$. We call this $\pi_{E}$ the almost monodromy quasi-representation of $E$.

This definition makes sense because of the estimate

$$
\left\|\Gamma_{\gamma}^{\nabla}-1\right\| \leq\left\|R^{E}\right\| \cdot \operatorname{Area}(D)
$$

for any loop $\gamma$ bounded by a surface $D$, i.e., $\gamma=\partial D \subset M$ (we refer to Gro96, Section $\left.4 \frac{1}{4}\right]$ ). We call this quasi-representation as the almost monodromy quasirepresentation of $E$.

Hereafter we focus on the torus $M=V / \Pi$ equipped with the standard Riemannian metric. We fix $\ell_{j} \in \Omega M$ as the geodesic loop in $v_{j}$-direction starting from the origin $0 \in V / \Pi$. Note that $\ell_{j}$ represents $t_{j} \in \pi_{1}(M)$. In this case $U_{j}:=\pi_{E}\left(t_{j}\right)$ satisfies $\left\|\left[U_{j}, U_{l}\right]\right\|<\left\|R_{E}\right\|$ since the loop $\ell_{l}^{-1} \circ \ell_{j}^{-1} \circ \ell_{l} \circ \ell_{j}$ is bounded by a standard square, which has area 1.

2.2. Gromov-Lawson construction. Gromov-Lawson [GL83] gives a systematic construction of almost flat bundles using the topology, i.e., enlargeability, of the base space. Here we review their construction by focusing on the case that the base space $M$ is a torus.

Let $\kappa_{a}: V \rightarrow V$ denote scaling map by $a$, that is, $\kappa_{a}(v)=a v$ for any $v \in V$. Since $\kappa_{a}(N \Pi)=\Pi, \kappa_{a}$ induces a continuous map

$$
\kappa_{a}: V / N \Pi \rightarrow V / \Pi \text {. }
$$

Note that $\kappa_{a}$ is $a^{2}$-area contracting, that is, the induced bundle map

$$
d \kappa_{a}: \bigwedge^{2} T M \rightarrow \bigwedge^{2} T M
$$

satisfies $d \kappa_{a}=a^{2}$. Hence we have

$$
\left\|R_{\kappa_{a}^{*} E}\right\|=a^{2}\left\|R_{E}\right\| .
$$

Let $q_{a}: V / N \Pi \rightarrow V / \Pi$ denote the $N^{n}$-fold covering map. Then the push-forward bundle

$$
E_{N}:=q_{a} ! \kappa_{a}^{*} E=\bigsqcup_{x \in V / \Pi} \bigoplus_{q(\bar{x})=x}\left(\kappa_{a}^{*} E\right)_{\bar{x}}
$$

has a connection induced from that of $\kappa_{a}^{*} E$.

Lemma 2.2. For any vector bundle $E$ on $V / \Pi$, we have

$$
\operatorname{Index}\left(D^{E_{N}}\right)=\operatorname{Index}\left(D^{E}\right) .
$$

Proof. By definition $q$ ! extends to a unitary

$$
q_{a !}: L^{2}\left(V / N \Pi, \kappa_{a}^{*} E\right) \rightarrow L^{2}\left(V / \Pi, q_{a !} \kappa_{a}^{*} E\right) .
$$

Also, the conformal transformation $\kappa_{a}$ gives rise to a unitary

$$
\kappa_{a}^{*}: L^{2}(V / \Pi, E) \rightarrow L^{2}\left(V / N \Pi, \kappa_{a}^{*} E\right)
$$


determined by $\kappa_{a}^{*}(\xi)(x)=\frac{1}{a^{d / 2}} \xi\left(\kappa_{a}(x)\right)$. Now the diagram

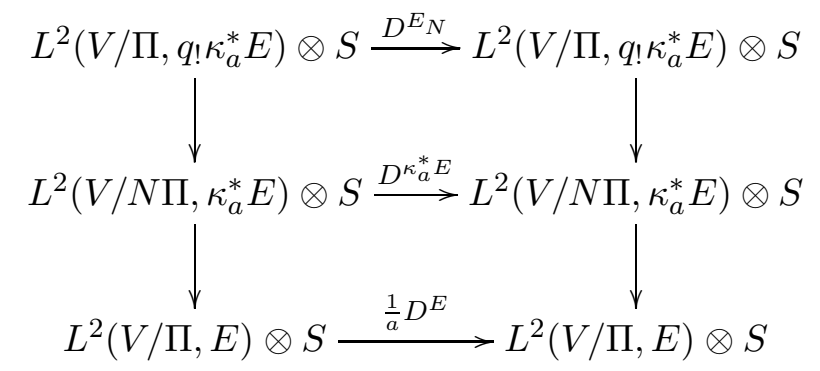

commutes, and hence $\operatorname{Index}\left(D^{E_{N}}\right)=\operatorname{Index}\left(\frac{1}{a} D^{E}\right)=\operatorname{Index}\left(D^{E}\right)$ holds.

2.3. Lattice covariant derivative as almost monodromy. Finally we observe that the lattice covariant derivatives $U_{j}^{a, E}$ introduced in Section 1 is nothing but the almost monodromy quasi-representation of the almost flat bundle obtained by the Gromov-Lawson construction.

Let $\mathbf{v}_{1}, \cdots, \mathbf{v}_{n}$ denote the standard basis of the vector space $V$. Let $\ell_{j}$ denote the image of the affine segment $\left[0, \mathbf{v}_{j}\right] \subset V$ to $V / \Pi$, which is a closed loop representing $t_{j} \in \pi_{1}(V / \Pi)$. Let $\pi_{a, E}$ denote the corresponding almost monodromy quasi-representation of the bundle $q ! \kappa_{a}^{*} E$ in the sense of Definition 2.1. Then it is a $\left(\left\|R_{E}\right\| a^{2}, \mathcal{G}\right)$-representation of $\Pi$.

Lemma 2.3. The $\left(a^{2}\left\|R_{E}\right\|, \mathcal{G}\right)$-representation $\pi_{a, E}$ satisfies $\pi_{a, E}\left(t_{j}\right)=U_{j}^{a, E}$.

Proof. The fiber of $E_{N}=q_{a} ! \kappa_{a}^{*} E$ at $0 \in V / \Pi$ is identified with the direct sum

$$
\bigoplus_{q_{a}(x)=0}\left(\kappa_{a}^{*} E\right)_{x}=\bigoplus_{x \in \Pi / N \Pi}\left(\kappa_{a}^{*} E\right)_{x}=\bigoplus_{x \in a \Pi / \Pi} E_{x} .
$$

The connection on $E_{N}$ is imposed from that of $E$ in the way that the parallel transport $\Gamma_{\gamma}^{E_{N}}$ along a path $\gamma:[0,1] \rightarrow V / \Pi$ is written as

$$
\begin{aligned}
\Gamma_{\gamma}^{E_{N}} & =\bigoplus_{q_{a}(\tilde{\gamma})=\gamma}\left(\Gamma_{\tilde{\gamma}}^{\kappa_{a}^{*} E}: \kappa_{a}^{*} E_{\tilde{\gamma}(0)} \rightarrow \kappa_{a}^{*} E_{\tilde{\gamma}(1)}\right) \\
& =\bigoplus_{q_{a}(\tilde{\gamma})=\gamma}\left(\Gamma_{\kappa_{a} \circ \tilde{\gamma}}^{E}: E_{\kappa_{a}(\tilde{\gamma}(0))} \rightarrow E_{\kappa_{a}(\tilde{\gamma}(1))}\right) .
\end{aligned}
$$

In particular we have

$$
\begin{aligned}
\pi_{a, E}\left(t_{j}\right)=\Gamma_{\left[0, \mathbf{v}_{j}\right]}^{E_{N}} & =\bigoplus_{x \in \Pi / N \Pi}\left(\Gamma_{\left[x, x+\mathbf{v}_{j}\right]}^{\kappa_{a}^{*} E}:\left(\kappa_{a}^{*} E\right)_{x} \rightarrow\left(\kappa_{a}^{*} E\right)_{x+\mathbf{v}_{j}}\right) \\
& =\bigoplus_{x \in a \Pi / \Pi}\left(\Gamma_{\left[x, x+a \mathbf{v}_{j}\right]}^{E}: E_{x} \rightarrow E_{x+a \mathbf{v}_{j}}\right) .
\end{aligned}
$$

The right hand side is the same as the operator $U_{j}^{a, E}$ defined in (1.2).

\section{K-THEORY OF THE UNIVERSAL HERMITIAN WILSON-DiRAC OPERATOR}

In the last section we observe that the shift operators $U_{j}^{a, E}$ are viewed as the image of $t_{j} \in \Pi$ under the quasi-representation $\pi_{a, E}$. This fact suggests that the lattice Wilson-Dirac operator is also viewed as the image of the 'universal' element lying in the group algebra $\mathbb{C}[\Pi]$, or its $\mathrm{C}^{*}$-algebra completion. In this section we 
describe the universal Wilson-Dirac operator as a self-adjoint element of a $\mathrm{C}^{*_{-}}$ algebra. This relates the invariant $\mathbf{I}\left(D_{W}^{a, E}+m \gamma\right)$ introduced in Definition 1.4 with the Bott element of the $\mathrm{C}^{*}$-algebra K-group $\mathrm{K}_{0}\left(C^{*} \Pi\right)$.

3.1. Universal hermitian Wilson-Dirac operator. Let $\hat{\Pi}$ denote the Pontrjagin dual $\operatorname{Hom}(\Pi, \mathbb{T})$ of $\Pi$. According to the Gelfand-Naimark duality, the group $C^{*}$ algebra $C^{*} \Pi$ is isomorphic to the $\mathrm{C}^{*}$-algebra $C(\hat{\Pi})$ of continuous functions on $\Pi$ by regarding $t \in \Pi$ as the continuous function $\chi \mapsto \chi(t)$.

Definition 3.1. We define the universal hermitian Wilson-Dirac operator $\hat{D}_{W} \in$ $C^{*} \Pi \otimes \operatorname{End}(S)$ as $\hat{U}_{j}:=t_{j}, \hat{\nabla}_{j}:=\hat{U}_{j}-1$ and

$$
\begin{aligned}
\hat{D} & :=\sum c\left(v_{j}\right)\left(\hat{\nabla}_{j}-\hat{\nabla}_{j}^{*}\right) / 2, \\
\hat{W} & :=\sum\left(\hat{\nabla}_{j}+\hat{\nabla}_{j}^{*}\right) / 2, \\
\hat{D}_{W} & :=\hat{D}+\gamma \hat{W} .
\end{aligned}
$$

Let $U_{1}, \cdots, U_{d}$ be a $d$-tuple of mutually $\varepsilon$-commuting unitaries on a (finite rank) Hilbert space $\mathcal{H}$ and let $\pi$ denotes the $(\varepsilon, \mathcal{G})$-representation of $\Pi$ determined by $\pi\left(t_{j}\right):=U_{j}$ and $\pi\left(t_{j}^{ \pm 1} t_{l}^{ \pm 1}\right):=U_{j}^{ \pm 1} U_{l}^{ \pm 1}$. We extend $\pi$ to a linear map $\pi: \mathbb{C}[\Pi] \rightarrow$ $\mathbb{B}(\mathcal{H})$ by

$$
\pi\left(t_{1}^{k_{1}} \cdots t_{d}^{k_{d}}\right):=\pi\left(t_{1}\right)^{k_{1}} \cdots \pi^{\mathrm{b}}\left(t_{d}\right)^{k_{d}} .
$$

Then we can associate to $\pi$ a matrix

$$
\pi\left(\hat{D}_{W}\right)=\sum_{j=1}^{n} \frac{1}{2}\left(U_{j}-U_{j}^{*}\right) \cdot c\left(v_{j}\right)+\sum_{j=1}^{n}\left(\frac{1}{2}\left(U_{j}+U_{j}^{*}\right)-1\right) \cdot \gamma .
$$

By comparing the above definitions with Definition 1.3 , Lemma2.3 is rephrased as the following lemma.

Lemma 3.4. The hermitian Wilson-Dirac operator $D_{W}^{a, E}$ coincides with $\frac{1}{a} \pi_{a, E}\left(\hat{D}_{W}\right)$.

Since the elements $\hat{U}_{j}$ and $\hat{U}_{l}$ are commutative, the spectral theory of the square $\left(\hat{D}_{W}+m \gamma\right)^{2}$ of the massive universal hermitian Wilson-Dirac operator is much easier than analysing Wilson-Dirac operators itself.

Lemma 3.5. For $0<m<2$, the massive universal hermitian Wilson-Dirac operator $\hat{D}_{W}+m \gamma$ is invertible.

Proof. Here we write as $t_{j}=e^{2 \pi i k_{j}}$, where $k_{j}$ is the $j$-th coordinate of $\hat{\Pi} \cong(\mathbb{R} / \mathbb{Z})^{d}$. Through the identification $C^{*} \Pi \cong C(\hat{\Pi})$, the operators $\hat{D}$ and $\hat{W}$ are identified with $\operatorname{End}(S)$-valued functions on $\hat{\Pi}$ as

$$
\hat{D}=\sum_{j=1}^{d} c\left(v_{j}\right) \cdot i \sin \left(2 \pi k_{j}\right), \quad \hat{W}=\sum_{j=1}^{d}\left(\cos \left(2 \pi k_{j}\right)-1\right) .
$$

Hence we have

$$
\begin{aligned}
& \left(\hat{D}_{W}+m \gamma\right)^{2}=\hat{D}^{2}+(\hat{W}+m)^{2} \\
= & \sum_{j} \sin \left(2 \pi k_{j}\right)^{2}+\left(\left(\sum_{j} \cos \left(2 \pi k_{j}\right)-1\right)+m\right)^{2} \geq 0 .
\end{aligned}
$$


The first and second components are both non-negative and never be zero simultaneously. Therefore $\left(\hat{D}_{W}+m \gamma\right)^{2}>0$ holds, i.e., the matrix-valued function $\hat{D}_{W}+m \gamma$ is invertible.

3.2. K-theory class of the universal Wilson-Dirac operator. Let $A$ be a $\mathrm{C}^{*}$ algebra. If $A$ is unital, i.e., $1 \in A$, its $\mathrm{K}_{0}$-group $\mathrm{K}_{0}(A)$ is defined as the set of homotopy classes of projections in $\mathbb{M}_{n}(A)$ with the summation given by the direct sum (for a foundation of $\mathrm{C}^{*}$-algebra K-theory, we refer to [RLL00]). We remark that the space of self-adjoint and invertible operators in a $\mathrm{C}^{*}$-algebra $A$ is homotopy equivalent to the space of projections by the continuous map $h \mapsto(h /|h|+1) / 2$, with the homotopy inverse $p \mapsto 2 p-1$. Hereafter, for a self-adjoint operator $h \in \mathbb{M}_{n}(A)$ we simply write $[h]$ for the corresponding element of the $\mathrm{K}_{0}$-group $[(h /|h|+1) / 2] \in \mathrm{K}_{0}(A)$.

By the Serre-Swan theorem, the $\mathrm{K}_{0}$-group $\mathrm{K}_{0}(C(X))$ of the commutative $\mathrm{C}^{*-}$ algebra $C(X)$ of continuous functions on a compact space $X$ is isomorphic to the topological K-group $\mathrm{K}^{0}(X)$. Hence the group $\mathrm{K}_{0}\left(C^{*} \Pi\right) \cong \mathrm{K}^{0}(\hat{\Pi})$ contains the Bott element $\beta$ of top degree, i.e., the image of the generator of $\mathrm{K}^{0}\left(\mathbb{D}^{n}, S^{n-1}\right) \cong \mathbb{Z}$ to $\mathrm{K}^{0}(\hat{\Pi})$ with respect to an open embedding.

Lemma 3.6. Let $c(v)$ and $\gamma$ be elements of $\mathbb{C} \ell_{d} \cong \operatorname{End}(S)$ as in Section $\square$ Set

$$
h:=\sum_{j=1}^{N} c\left(v_{j}\right) \cdot i x_{j}+\gamma x_{0}: S^{n} \rightarrow \operatorname{End}(S),
$$

where $x_{0}, \cdots, x_{n}$ is the standard coordinate of $S^{n} \subset \mathbb{R}^{n+1}$. Then $h$ is a selfadjoint matrix-valued function with $h^{2}=1$ and the Bott element $\beta \in \mathrm{K}^{0}\left(S^{n}\right)$ is represented by $h$ as $\beta=[h]-[\gamma]$.

Proof. This is understood from the viewpoint of Segal's connective K-theory [Seg77]. Let $F_{d}(n)$ denote the set of mutually commuting $d+1$-tuple $\left(A_{0}, A_{1}, \cdots, A_{d}\right)$ of $n \times n$ matrices such that $\sum A_{i}^{2}=1$ and set $F_{d}(\infty)=\bigcup_{n} F_{d}(n)$ with respect to the inclusion $\left(A_{0}, A_{1}, \cdots, A_{n}\right) \mapsto\left(A_{0} \oplus 1, A_{1} \oplus 0, \cdots, A_{d} \oplus 0\right)$. Similarly, let $\Phi_{d}(n)$ denote the set of self-adjoint unitaries on $S \otimes \mathbb{C}^{n}$ and set $\Phi_{d}(\infty)=\bigcup_{n} \Phi_{d}(n)$ with respect to $B \mapsto B \oplus \gamma$. Then the map $\varphi: F_{d}(\infty) \rightarrow \Phi_{d}(\infty)$ given by $\left(A_{0}, A_{1}, \cdots, A_{d}\right) \mapsto A_{0} \gamma+\sum i A_{j} c\left(v_{j}\right)$ induces an isomorphism of the $\pi_{d}$-group ([Seg77, Proposition 1.3]). Moreover, the map $c: F_{d} \rightarrow P_{d}$, where $P_{d}$ is the configuration space of points in $S^{d}$ (in other words, $P_{d}=\mathrm{SP}^{\infty}\left(S^{d}\right)$ ), sending $\left(A_{0}, A_{1}, \cdots, A_{d}\right)$ to its joint spectrum, also induces an isomorphism of the $\pi_{d^{-}}$ group ([Kub16a, Proposition 3.4]). Now the map $\mathbf{x}:=\left(x_{0}, x_{1}, \cdots, x_{d}\right): S^{d} \rightarrow$ $F_{d}(1)$ satisfies $\varphi \circ \mathbf{x}=h$ and $[c \circ \mathbf{x}] \in\left[S^{d}, P_{d}\right] \cong H^{d}\left(S^{d}\right) \cong \mathbb{Z}$ is the generator. This shows the lemma.

As is proved in Lemma 3.5, the massive hermitian Wilson-Dirac operator $\hat{D}_{W}+$ $m \gamma$ is a self-adjoint invertible element of the $\mathrm{C}^{*}$-algebra $C^{*} \Pi \otimes \operatorname{End}(S)$, and hence it determines an element $\left[\hat{D}_{W}+m \gamma\right] \in \mathrm{K}_{0}\left(C^{*} \Pi\right) \cong \mathrm{K}^{0}(\hat{\Pi})$.

Proposition 3.7. For any $0<m<2$, the K-theory class $\left[\hat{D}_{W}+m \gamma\right] \in$ $\mathrm{K}_{0}\left(C^{*} \Pi\right) \cong \mathrm{K}^{0}(\hat{\Pi})$ satisfies

$$
\left[\hat{D}_{W}+m \gamma\right]-[\gamma]=\beta .
$$


Proof. Recall the identification of $\hat{D}_{W}$ with an $\operatorname{End}(S)$-valued continuous function on $\hat{\Pi}$ as in the proof of Lemma 3.5 , Let

$$
f:=\left(\sum_{j} \sin \left(2 \pi k_{j}\right)^{2}+\left(\left(\sum_{j} \cos \left(2 \pi k_{j}\right)-1\right)+m\right)^{2}\right)^{1 / 2}
$$

which is a positive function on $\hat{\Pi}$. In the space of self-adjoint invertible operatorvalued functions, $\hat{D}_{W}+m \gamma$ is homotopic to

$$
\frac{\hat{D}_{W}+m \gamma}{f}=\sum c\left(v_{j}\right) \frac{i \sin \left(2 \pi k_{j}\right)}{f}+\gamma \frac{\left(\sum_{j}\left(\cos \left(2 \pi k_{j}\right)-1\right)\right)+m}{f}=F^{*} h,
$$

where $F: \hat{\Pi} \rightarrow S^{d}$ is defined as

$$
F(\mathbf{k})=\left(\frac{\left(\sum_{j}\left(\cos \left(2 \pi k_{j}\right)-1\right)\right)+m}{f(\mathbf{k})} \frac{\sin \left(2 \pi k_{1}\right)}{f(\mathbf{k})}, \cdots \frac{\sin \left(2 \pi k_{d}\right)}{f(\mathbf{k})}\right)
$$

for $\mathbf{k}=\left(k_{1}, \cdots, k_{d}\right) \in \hat{\Pi}$. Since $(1,0, \cdots, 0)$ is the regular value of $F$ and $F^{-1}(1,0, \cdots, 0)=\{(0, \cdots, 0)\}$, the degree of $F$ is 1 and hence

$$
\left[\hat{D}_{W}+m \gamma\right]-[\gamma]=F^{*}([h]-[\gamma])=\beta \in \mathrm{K}^{0}(\hat{\Pi})
$$

by Lemma 3.6

Remark 3.8. The invertible matrix-valued function $\hat{D}_{W}+m \gamma$ and its K-theory class in $\mathrm{K}^{0}(\hat{\Pi})$, particularly when $d=0,1,2,3$, is also known as a model of Chern insulators in the theory of topological insulators in condensed-matter physics (see for example [PS16, Section 2.2.4]).

3.3. Approximate $*$-homomorphism and K-theory. For a unital $\mathrm{C}^{*}$-algebra $A$, a unitary representation $\pi: \Pi \rightarrow \mathcal{U}(A)$ extends to a $*$-homomorphism $C^{*} \Pi \rightarrow A$. Although a single quasi-representation does not necessarily extend to a continuous map on $C^{*} \Pi$, a nice collection $\pi_{n}$ of quasi-representations is able to be treated as a single $*$-homomorphism in the following way.

Let $\pi_{n}: \mathcal{G} \rightarrow \mathcal{U}\left(\mathcal{H}_{n}\right)$ be a sequence of finite rank $\left(\varepsilon_{n}, \mathcal{G}\right)$-representations such that $\varepsilon_{n} \rightarrow 0$. We pick a collection of embeddings $\mathcal{H}_{n} \subset \mathcal{H}$ into a single separable infinite dimensional Hilbert space $\mathcal{H}$. We simply write $\mathbb{K}$ for the compact operator algebra $\mathbb{K}(\mathcal{H})$. Let us consider the quotient $\mathrm{C}^{*}$-algebra

$$
\mathcal{Q}:=\frac{\prod_{n \in \mathbb{N}} \mathbb{K}}{\bigoplus_{n \in \mathbb{N}} \mathbb{K}}
$$

For $\left(a_{n}\right)_{n \in \mathbb{N}} \in \prod_{\mathbb{N}} \mathbb{K}$, we write $\left(a_{n}\right)_{n \in \mathbb{N}}^{b}$ for its image in $\mathcal{Q}$. denote the projection. Then

$$
\pi^{b}\left(t_{j}\right):=\left(\pi_{n}\left(t_{j}\right) \oplus 1_{\mathcal{H}_{n}^{\perp}}\right)_{n \in \mathbb{N}}^{b}
$$

satisfies $\left[\pi^{b}\left(t_{j}\right), \pi^{b}\left(t_{l}\right)\right]=0$. That is, $\pi^{b}$ extends to a unitary representation of $\Pi$ by $\pi^{\mathrm{b}}\left(t_{1}^{k_{1}} \cdots t_{d}^{k_{d}}\right):=\pi^{\mathrm{b}}\left(t_{1}\right)^{k_{1}} \cdots \pi^{\mathrm{b}}\left(t_{d}\right)^{k_{d}}$, and hence we obtain a $*$-homomorphism

$$
\pi^{b}: C^{*} \Pi \rightarrow \mathcal{Q} .
$$

Lemma 3.10. The $\mathrm{K}_{0}$-group of $C^{*}$-algebras $\bigoplus_{\mathbb{N}} \mathbb{K}, \prod_{\mathbb{N}} \mathbb{K}$ and $\mathcal{Q}$ are

$$
\mathrm{K}_{0}\left(\bigoplus_{\mathbb{N}} \mathbb{K}\right) \cong \bigoplus_{\mathbb{N}} \mathbb{Z}, \quad \mathrm{K}_{0}\left(\prod_{\mathbb{N}} \mathbb{K}\right) \cong \prod_{\mathbb{N}} \mathbb{Z}, \quad \mathrm{K}_{0}(\mathcal{Q}) \cong \frac{\prod_{\mathbb{N}} \mathbb{Z}}{\bigoplus_{\mathbb{N}} \mathbb{Z}}
$$

respectively. 
Proof. The first and second isomorphisms are straightforward from the definition of $\mathrm{C}^{*}$-algebra K-theory. The third is a consequence of the six-term exact sequence associated to the $\mathrm{C}^{*}$-algebra extension $0 \rightarrow \bigoplus_{\mathbb{N}} \mathbb{K} \rightarrow \prod_{\mathbb{N}} \mathbb{K} \rightarrow \mathcal{Q} \rightarrow 0$.

Hereafter, for a sequence $\left(k_{n}\right)_{n \in \mathbb{N}}$ of integers, we write $\left(k_{n}\right)_{n \in \mathbb{N}}^{b}$ for its under the quotient $\prod_{\mathbb{N}} \mathbb{Z} \rightarrow \prod_{\mathbb{N}} \mathbb{Z} / \bigoplus_{\mathbb{N}} \mathbb{Z}$.

By the above lemma, $\pi^{b}$ induces a group homomorphism

$$
\pi^{b}: \mathrm{K}_{0}\left(C^{*} \Pi\right) \rightarrow \mathrm{K}_{0}(\mathcal{Q}) \cong \frac{\prod_{\mathbb{N}} \mathbb{Z}}{\bigoplus_{\mathbb{N}} \mathbb{Z}}
$$

In other words,

$$
\left(\pi_{n}\right)_{n \in \mathbb{N}} \mapsto \pi^{b}(\beta) \in \frac{\prod_{\mathbb{N}} \mathbb{Z}}{\bigoplus_{\mathbb{N}} \mathbb{Z}}
$$

is a topological invariant for sequences of $\left(\varepsilon_{n}, \mathcal{G}\right)$-representations of $\Pi$ with $\varepsilon_{n} \rightarrow$ 0 . It is trivial if $\left(\pi_{n}\right)_{n \in \mathbb{N}}$ is homotopic to a representation, i.e., $\pi^{b}$ lifts to a $*$-homomorphism $C^{*} \Pi \rightarrow \prod_{\mathbb{N}} \mathbb{K}$ since any $*$-homomorphism $C(\hat{\Pi}) \rightarrow \mathbb{K}$ is homotopic to the trivial one.

Now we apply Proposition 3.7 to get an explicit description of the invariant (3.11).

Lemma 3.12. Let $\lambda$ denote the bottom of the spectrum of $\left(\hat{D}_{W}+m \gamma\right)^{2}$ and let $0<C<\lambda$. Then there is a constant $\varepsilon>0$ such that $\pi\left(\hat{D}_{W}+m \gamma\right)^{2}>C$ holds for any $(\varepsilon, \mathcal{G})$-representation $\pi$ of $\Pi$.

Instead of an analytic proof using norm estimates (as is given in Proposition 4.3 later), we prove this lemma by using an abstract spectral theory of $\mathrm{C}^{*}$-algebras.

Proof. Let us choose $C^{\prime}$ with $C<C^{\prime}<\lambda$ and we show that $\pi_{n}\left(\hat{D}_{W}+m \gamma\right)^{2} \geq C^{\prime}$ except for finitely many $n$ 's. To this end, we assume the contrary, i.e., for any $n \in \mathbb{N}$ there is a $\left(\frac{1}{n}, \mathcal{G}\right)$-representation $\pi_{n}$ of $\Pi$ such that $\pi\left(\hat{D}_{W}+m \gamma\right)^{2}-C^{\prime}$ is not positive. We bundle these $\pi_{n}$ 's to get a $*$-homomorphism $\pi^{b}$ as in (3.9). Then the spectrum $\sigma\left(\pi^{b}\left(\hat{D}_{W}^{2}+m \gamma\right)^{2}-C^{\prime}\right)$ is the set of accumulation points of $\sigma\left(\pi_{n}\left(\hat{D}_{W}+m \gamma\right)^{2}-C^{\prime}\right)$, and hence it intersects with $\left[-C^{\prime}, 0\right]$ non-trivially. This contradicts with

$$
\begin{aligned}
\sigma\left(\pi^{b}\left(\hat{D}_{W}+m \gamma\right)^{2}-C^{\prime}\right) & =\sigma\left(\pi^{b}\left(\left(\hat{D}_{W}+m \gamma\right)^{2}-C^{\prime}\right)\right) \\
& \subset \sigma\left(\left(\hat{D}_{W}+m \gamma\right)^{2}-C^{\prime}\right) \subset\left[\lambda-C^{\prime}, \infty\right)
\end{aligned}
$$

which is a standard fact in the spectral theory of $\mathrm{C}^{*}$-algebras.

This lemma shows that

$$
\mathbf{I}\left(\pi\left(\hat{D}_{W}\right)+m \gamma\right) \in \mathbb{Z}
$$

is a topological obstruction for $\pi$ to be homotopic to a representation of $\Pi$ in the space of $(\varepsilon, \mathcal{G})$-commuting matrices for sufficiently small $\varepsilon>0$.

Theorem 3.14. Let $\pi_{n}$ be a sequence of $\left(\varepsilon_{n}, \mathcal{G}\right)$-representations of $\Pi$ with $\varepsilon_{n} \rightarrow 0$ such that $\pi_{n}\left(\hat{D}_{W}+m \gamma\right)$ is invertible for $n \geq n_{0}$. Then we have

$$
\pi^{b}(\beta)=\left(\mathbf{I}\left(\pi_{n}\left(\hat{D}_{W}\right)+m \gamma\right)\right)_{n \geq n_{0}}^{b} \in \frac{\prod \mathbb{Z}}{\bigoplus \mathbb{Z}} .
$$


Here we write $(\cdot)_{n \geq n_{0}}^{b}$ for the image under the compositions $\prod_{n \geq n_{0}} \mathbb{K} \rightarrow$ $\prod_{n \in \mathbb{N}} \mathbb{K} \rightarrow \mathcal{Q}$ or $\prod_{n \geq n_{0}} \mathbb{Z} \rightarrow \prod_{n \in \mathbb{N}} \mathbb{Z} \rightarrow \prod_{\mathbb{N}} \mathbb{Z} / \bigoplus_{\mathbb{N}} \mathbb{Z}$

Proof. The identification $\mathrm{K}_{0}(\mathcal{Q}) \cong \prod_{\mathbb{N}} \mathbb{Z} / \bigoplus_{\mathbb{N}} \mathbb{Z}$ is given by mapping $\left[\left(p_{n}\right)_{n \in \mathbb{N}}^{b}\right] \in$ $\mathrm{K}_{0}(\mathcal{Q})$ to $\left(\operatorname{rank} p_{n}\right)_{n \in \mathbb{N}^{b}}^{b}$ Hence, for an invertible element $\left(h_{n}\right)_{n \in \mathbb{N}}^{b} \in \mathcal{Q}$, its Ktheory class $\left[\left(h_{n}\right)_{n \in \mathbb{N}}^{b}\right] \in \mathrm{K}_{0}(\mathcal{Q})$ corresponds to $\left(\operatorname{dim} E_{>0}\left(h_{n}\right)\right)_{n \in \mathbb{N}}^{b}$.

By Proposition 3.7 we have

$$
\begin{aligned}
\pi^{b}(\beta) & =\left[\pi^{b}\left(\hat{D}_{W}+m \gamma\right)\right]-[\gamma] \\
& =\left(\left[\left(\pi_{n}\left(\hat{D}_{W}+m \gamma\right)\right)_{n \geq n_{0}}^{b}\right]-[\gamma] .\right.
\end{aligned}
$$

Through the identification as above, the right hand side corresponds to

$$
\left(\operatorname{dim} E_{>0}\left(\pi_{n}\left(\hat{D}_{W}+m \gamma\right)\right)-\operatorname{dim} E_{>0}(\gamma)\right)_{n \in \mathbb{N}}^{b}=\left(\mathbf{I}\left(\pi_{n}\left(\hat{D}_{W}\right)+m \gamma\right)\right)_{n \geq n_{0}}^{b}
$$

by Reamrk 1.5 and Lemma 3.4 ,

Remark 3.15. When $d=2$, this invariant is the same as the one given in [EL91]. Our description using the Wilson-Dirac operator is efficient in computation since there is no need to calculate the functional calculus of a large matrix.

\section{Wilson-Dirac INDEX THEOREM AS ALMOST FLAT INDEX PAIRING}

In this section we give a proof of Theorem 1.6 as an application of the HankeSchick index pairing [HS06].

4.1. Higher index pairing. The index pairing is the pairing between the K-theory and K-homology groups of a manifold $M$ (a reference is [HR00]). It assigns to $[\mathcal{E}] \in \mathrm{K}^{0}(M)$ and $[\mathcal{D}] \in \mathrm{K}_{0}(M)$, where $\mathcal{E}$ is a complex vector bundle and $\mathcal{D}$ is an elliptic operator on $M$, the Fredholm index Index $\mathcal{D}^{\mathcal{E}}$ of the twisted operator $\mathcal{D}^{\mathcal{E}}$. More generally, a K-homology element $[\mathcal{D}] \in \mathrm{K}_{0}(M)$ induces the "index pairing with coefficient", i.e., a homomorphism

$$
\langle\sqcup,[\mathcal{D}]\rangle_{A}: \mathrm{K}_{0}(C(M) \otimes A) \rightarrow \mathrm{K}_{0}(A) .
$$

This homomorphism is described as following. Let $\mathcal{H}$ be a Hilbert space equipped with a $*$-representation $C(M) \rightarrow \mathbb{B}(\mathcal{H})$. A K-homology element in the Atiyah-Kasparov picture (see for example [HR00, Definition 8.1.1]) is represented by a bounded operator $F \in \mathbb{B}(\mathcal{H})$ such that $[F, f], F^{*} F-1, F F^{*}-1$ are compact operators for any $f \in C(M)$. Our concern is the Dirac fundamental class $[D]$ of $M$ represented by $\mathcal{H}:=L^{2}\left(M, S^{+}\right) \cong L^{2}\left(M, S^{-}\right)$(this unitary isomorphism is given by a fixed Borel isomorphism of vector bundles $S^{+} \cong S^{-}$) and

$$
F:=D\left(1+D^{*} D\right)^{-1 / 2}: L^{2}\left(M, S^{+}\right) \rightarrow L^{2}\left(M, S^{-}\right) .
$$

Another ingredient of the proof is the Mishchenko bundle $\mathcal{L}:=\tilde{M} \times_{\Pi} C^{*} \Pi$, where $\Pi$ acts on $C^{*} \Pi$ by the multiplication from the left. This is a flat bundle of Hilbert $C^{*} \Pi$-modules. Note that the Serre-Swan theorem with coefficient also holds; the group $\mathrm{K}_{0}(C(M) \otimes A)$ is isomorphic to the Grothendieck group of the semigroup of bundles of finitely generated projective Hilbert $A$-modules (see for example [Sch05, Subsection 3.2]). Therefore, $\mathcal{L}$ determines an element $[\mathcal{L}] \in \mathrm{K}_{0}\left(C(M) \otimes C^{*} \Pi\right)$.

Remark 4.1. Here we enumerate some basic facts on the index pairing and the Mishchenko bundle which will be used in the proof of Theorem 1.6 . 
(1) The index pairing with coefficient in $\mathbb{C}$ is the same thing as the usual index pairing, i.e., $\langle[E],[D]\rangle_{\mathbb{C}}=\operatorname{Index}\left(D^{E}\right) \in \mathrm{K}_{0}(\mathbb{C}) \cong \mathbb{Z}$ (see for example [HR00, Proposition 4.8.10 (c)]).

(2) The index pairing is compatible with the base-change: Let $\phi: A \rightarrow B$ be a $*$-homomorphism. Then we have

$$
\left\langle\left(\operatorname{id}_{C(M)} \otimes \phi\right)_{*}[p],[F]\right\rangle_{B}=\phi_{*}\left(\langle[p],[F]\rangle_{A}\right) .
$$

This holds by definition of the index pairing above because $\left(\operatorname{id}_{\mathbb{B}(\mathcal{H})} \otimes\right.$ $\phi)\left(F_{p}\right)=F_{\phi(p)}$ holds.

(3) The bundle $\mathcal{L}$ is universal among flat bundles of Hilbert $\mathrm{C}^{*}$-modules: If we have a flat bundle $\mathcal{E} \rightarrow M$ of Hilbert $A$-modules, the monodromy representation $\pi_{\mathcal{E}}: \Pi \rightarrow \mathbb{B}(E)$ (where $E$ is a typical fiber of $\mathcal{E}$ ) is associated. Then, by definition of $\mathcal{L}$, the base change $\mathcal{L} \otimes_{\pi_{E}} E$ is isomorphic to $\mathcal{E}$ itself. In the level of K-theory, we have

$$
\left(\operatorname{id}_{C(M)} \otimes \pi_{\mathcal{E}}\right)_{*}[\mathcal{L}]=[\mathcal{E}]
$$

(4) The higher index pairing $\langle[\mathcal{L}],[D]\rangle_{C^{*} \Pi} \in \mathrm{K}_{0}\left(C^{*} \Pi\right)$ is the Bott element $\beta$. This is essentially proved by Lusztig [Lus72] by using the Atiyah-Singer family index theorem. Indeed, through the identification $C^{*} \Pi \cong C(\hat{\Pi})$, the index pairing with $\mathcal{L}$ is identified with the family index for the fiberwise Dirac operator on the fiber bundle $M \times \hat{\Pi} \rightarrow \hat{\Pi}$ twisted by the Poincare line bundle

$$
\mathcal{P}:=V \times \hat{\Pi} \times \mathbb{C} /\left\{(v, \chi, \xi) \sim\left(v+t, \chi, \chi(t)^{*} \xi\right)\right\}
$$

Lemma 4.2 ([HS06, Theorem 3.8]). Let $\pi_{N}:=\pi_{a, E}$ be the quasi-representation of $\Pi$ defined in Subsection 2.3 and let $\pi^{b}$ be the $*$-homomorphism as in (3.9). Then we have

$$
\pi^{b}(\beta)=\left(\operatorname{Index} D^{E}\right)_{N \in \mathbb{N}}^{b} \in \frac{\prod \mathbb{Z}}{\bigoplus \mathbb{Z}}
$$

Proof. For the self-consistency of the paper, we quickly review the proof given in [HS06]. For each $N, \mathcal{E}_{N}:=E_{N} \otimes \mathcal{H}^{*}$ is a bundle of projective Hilbert $\mathbb{K}(\mathcal{H})$ modules which is identified with $E_{N}$ through the isomorphism $\mathrm{K}_{0}(C(M)) \cong$ $\left.\mathrm{K}_{0}(C(M) \otimes \mathbb{K}(\mathcal{H}))\right)$. The connection of $E_{N}$ extends to that of $\mathcal{E}_{N}$ whose curvature has the norm less than $a^{2}\left\|R_{E}\right\|$ (for the connection and curvature of Hilbert $\mathrm{C}^{*}$ module bundles, see [Sch05, Section 4]) . Let $\mathcal{E}:=\prod \mathcal{E}_{N}$, which is a Hilbert $\prod \mathbb{K}$-module. Then we have

$$
\langle[\mathcal{E}],[D]\rangle_{\prod \mathbb{K}}=\left(\operatorname{Index} D^{E_{N}}\right)_{N}=\left(\operatorname{Index}\left(D^{E}\right)\right)_{N} \in \mathrm{K}_{0}\left(\prod \mathbb{K}\right) \cong \prod \mathbb{Z}
$$

by Lemma2.2 and Remark 4.1(1). Moreover, the base-change $\mathcal{E} \otimes_{\varphi} \mathcal{Q}$ is isomorphic to the flat bundle $V \times{ }_{\pi^{b}} \mathcal{Q}$ associated to $\pi^{b}$. 
Let $\eta^{b}$ denote the quotient map $\prod \mathbb{K} \rightarrow \mathcal{Q}$. Now we obtain that

$$
\begin{aligned}
\pi_{*}^{b}(\beta) & =\pi_{*}^{b}\left(\langle[\mathcal{L}],[D]\rangle_{C^{*} \Pi}\right) \\
& =\left\langle\left(\operatorname{id}_{C(M)} \otimes \pi^{b}\right)_{*}[\mathcal{L}],[D]\right\rangle_{\mathcal{Q}} \\
& =\left\langle\left(\operatorname{id}_{C(M)} \otimes \eta^{b}\right)_{*}[\mathcal{E}],[D]\right\rangle_{\mathcal{Q}} \\
& =\eta_{*}^{b}\left(\langle[\mathcal{E}],[D]\rangle_{\Pi \mathbb{K}}\right) \\
& =\left(\operatorname{Index}\left(D^{E}\right)\right)_{N \in \mathbb{N}}^{b} \in \frac{\prod \mathbb{Z}}{\bigoplus \mathbb{Z}} .
\end{aligned}
$$

Here each of the first, second, third and forth equalities follows from (4), (2), (3), (2) of Remark 4.1 respectively.

4.2. Proof of the main theorem. The proof of Theorem 1.6 (1) is essentially finished by Lemma 3.12, Theorem 3.14 and Lemma 4.2. For the proof of (2), we need a quantitative refinement of Lemma 3.12.

The following norm estimate is communicated to the author by Mikio Furuta. A more conceptual description from the viewpoint of geometric analysis will appear in the forthcoming paper $\left[\mathrm{FFM}^{+}\right]$. A similar estimate is also studied by Neuberger [Neu00].

Proposition 4.3. Let $D_{\kappa, m}:=\kappa \pi_{a, E}\left(\hat{D}_{W}\right)+m \gamma$. For any $\kappa \in[m, 1 / a]$, the square $D_{\kappa, m}^{2}$ is bounded from below by $m^{2}-4 d^{2}\left\|R_{E}\right\|$.

Proof. In the proof, we write $a \sim_{\varepsilon} b$ for $\|a-b\|<\varepsilon$. Set

$$
\begin{aligned}
x_{j} & :=\frac{1}{2 i}\left(U_{j}^{a, E}-\left(U_{j}^{a, E}\right)^{*}\right), \\
y_{j} & :=\frac{1}{2}\left(U_{j}^{a, E}+\left(U_{j}^{a, E}\right)^{*}\right), \\
z_{j} & :=U_{j}^{a, E}-1 .
\end{aligned}
$$

Note that we have $1-y_{j}=\frac{1}{2} z_{j} z_{j}^{*}$ and $\left\|z_{j}\right\| \leq 2$. Now the square $D_{\kappa, m}^{2}$ is bounded from below as

$$
\begin{aligned}
& D_{\kappa, m}^{2}=\left(\kappa \sum_{j} c_{j} \cdot i x_{j}+\gamma\left(\kappa \sum_{j}\left(y_{j}-1\right)+m\right)\right)^{2} \\
& \sim_{\varepsilon_{1}} \kappa^{2} \sum_{j} x_{j}^{2}+\left(\kappa \sum_{j}\left(y_{j}-1\right)+m\right)^{2} \\
&=\kappa^{2} \sum_{j} x_{j}^{2}+\kappa^{2} \sum_{j}\left(y_{j}-1\right)^{2}+\sum_{j \neq l}\left(1-y_{j}\right)\left(1-y_{l}\right) \\
&+2 \kappa m \sum_{j}\left(y_{j}-1\right)+m^{2} \\
&= 2 \kappa(\kappa-m) \sum_{j}\left(1-y_{j}\right)+\kappa^{2} \sum_{j \neq l}\left(1-y_{j}\right)\left(1-y_{l}\right)+m^{2} \\
& \sim_{\varepsilon_{2}} \kappa(\kappa-m) \sum_{j} z_{j}^{*} z_{j}+\frac{\kappa^{2}}{4} \sum_{j \neq l} z_{j} z_{j}^{*} z_{l}^{*} z_{l}+m^{2} \geq m^{2},
\end{aligned}
$$


where $\varepsilon_{1}>0$ and $\varepsilon_{2}>0$ are defined as

$$
\begin{aligned}
& \left\|-\sum_{j<l} \kappa^{2} c_{j} c_{l}\left[x_{j}, x_{l}\right]+\sum_{j} c_{j} \gamma\left[\kappa \cdot i x_{j},\left(\kappa \sum_{l}\left(y_{l}-1\right)+m\right)\right]\right\| \\
\leq & \kappa^{2} \sum_{j, l}\left(\left\|\left[x_{j}, x_{l}\right]\right\|+\left\|\left[x_{j}, y_{l} \|\right) \leq \kappa^{2} \cdot 2 d^{2}\right\| R_{E}\left\|a^{2} \leq 2 d^{2}\right\| R_{E} \|=: \varepsilon_{1}\right.
\end{aligned}
$$

and

$$
\frac{\kappa^{2}}{4}\left\|\sum_{j \neq l}\left(z_{j}\left[z_{j}^{*}, z_{l}\right] z_{l}^{*}+z_{j} z_{l}\left[z_{j}^{*}, z_{l}^{*}\right]\right)\right\| \leq 2 d^{2} \kappa^{2}\left\|R_{E}\right\| a^{2} \leq 2 d^{2}\left\|R_{E}\right\|=: \varepsilon_{2} .
$$

This inequality implies that the spectrum of $D_{\kappa, m}^{2}$ is bounded below by $m^{2}-$ $4 d^{2}\left\|R_{E}\right\|$ if $m \leq \kappa \leq 1 / a$.

As a consequence of Proposition 4.3, we obtain that the self-adjoint matrices $\pi_{a, E}\left(m \hat{D}_{W}\right)+m \gamma$ and $D_{W}^{a, E}+m \gamma$ are homotopic in the space of invertible selfadjoint matrices for any $m>4 d^{2}\left\|R_{E}\right\|$. In particular we obtain

$$
\mathbf{I}\left(\pi_{a, E}\left(\hat{D}_{W}\right)+\gamma\right)=\mathbf{I}\left(D_{W}^{a, E}+m \gamma\right) .
$$

Proof of Theorem [1.6. By (4.4), it suffices to show that

$$
\mathbf{I}\left(\pi_{a, E}\left(\hat{D}_{W}\right)+\gamma\right)=\operatorname{Index}\left(D^{E}\right)
$$

for sufficiently small $a>0$. This follows from Theorem 3.14 and Lemma 4.2 ,

\section{Generalizations}

In this section we discuss two generalizations of Theorem 1.6, the family version and the real and Clifford equivariant version. They are also considered by Adams [Ada02b] and Fukaya et. al. $\left[\overline{\left.\mathrm{FFM}^{+}\right]}\right.$respectively. Since our method is $\mathrm{K}$-theoretic and does not rely on any evaluation such as integration of differential forms, the same proof also works for these generalized setting.

5.1. Family index. Let $X$ be a compact space and let $E$ be a vector bundle over $M \times X$, which is thought of as a family of vector bundles $E_{x}:=\left.E\right|_{M \times\{x\}}$ parametrized by $X$. Let $\left\{\nabla_{x}\right\}_{x \in X}$ be a smooth family of hermitian connections on $E_{x}$. Then the fiberwise Dirac operator $\mathfrak{D}^{E}(x):=D^{E_{x}}$ is a continuous function on $X$ taking value in Fredholm operators. Its family index determines an element of the topological K-group $\mathrm{K}^{0}(X)$.

Also, the Gromov-Lawson construction as in Section 2 provides a continuous family $\pi_{a, E, X}:=\left\{\pi_{a, E_{x}}\right\}_{x \in X}$ of quasi-representations of $\Pi$ parametrized by $X$. It gives rise to a $*$-homomorphism $\pi_{X}^{b}: C^{*} \Pi \rightarrow \mathcal{Q} \otimes C(X)$. Note that the K-group $\mathrm{K}_{0}(\mathcal{Q} \otimes C(X))$ is isomorphic to $\prod \mathrm{K}^{0}(X) / \bigoplus \mathrm{K}^{0}(X)$. The family Wilson-Dirac operator $\mathfrak{D}_{W}^{a, E}:=\pi_{X}^{b}\left(\hat{D}_{W}\right)$ is a continuous function from $X$ to the space of selfadjoint matrices such that $\mathfrak{D}_{W}^{a, E}+m \gamma$ is invertible. Then $E_{>0}\left(\mathfrak{D}_{W}^{a, E}+m \gamma\right)$ is a vector bundle on $X$, and

$$
\mathbf{I}_{X}\left(\mathfrak{D}_{W}^{a, E}+m \gamma\right):=\left[E_{>0}\left(\mathfrak{D}_{W}^{a, E}+\frac{m}{a} \gamma\right)\right]-\left[E_{>0}(\gamma)\right] \in \mathrm{K}^{0}(X)
$$

is defined.

Now the same proof shows that both Theorem 3.14 and Lemma 4.2 hold for this family version. Consequently we obtain the following generalization. 
Theorem 5.1. The following hold.

(1) For any $0<m<2$, there is $a_{0}=1 / N_{0}$ such that $\mathfrak{D}_{W}^{a, E}+\frac{m}{a}$ is invertible and $\mathbf{I}_{X}\left(\mathfrak{D}_{W}^{a, E}+\frac{m}{a} \gamma\right)=\operatorname{Index}_{X}\left(\mathfrak{D}^{E}\right)$ holds for any $0<a<a_{0}$.

(2) There is $m_{0}>0$ such that, for any $m>m_{0}$ there is $a_{0}=1 / N_{0}$ such that $\mathfrak{D}_{W}^{a, E}+m \gamma$ is invertible and $\mathbf{I}_{X}\left(\mathfrak{D}_{W}^{a, E}+m \gamma\right)=\operatorname{Index}_{X}\left(\mathfrak{D}^{E}\right)$ holds for any $0<a<a_{0}$.

5.2. Real and Clifford-equivariant index. Here we consider the index theorem for real vector bundles on the torus $M$ with an arbitrary dimension. Throughout this subsection $C \ell_{p, q}$ denotes the Clifford algebra generated by $e_{1}, \cdots, e_{p}$ and $f_{1}, \cdots, f_{q}$ with $e_{j}^{2}=1$ and $f_{j}^{2}=-1$. Let $M$ be the $d$-dimensional standard torus and let $\mathcal{S}$ be the unique irreducible representation of the Clifford algebra $C \ell(V \oplus-V) \cong C \ell_{d, d}$. The $C \ell_{d, 0}$-Dirac operator

$$
\mathcal{D}:=\sum_{j=1}^{n} f_{j} \nabla_{v_{j}}: \Gamma(M, \mathcal{S}) \rightarrow \Gamma(M, \mathcal{S})
$$

is an odd self-adjoint operator anticommuting with the Clifford generators $e_{j}$ of $C \ell_{d}$. We write $\mathcal{D}^{E}$ for the corresponding twisted $C \ell_{d, 0}$-Dirac operator. In a similar way as in [AS69, Section 5], its Clifford index is defined as

$$
\operatorname{Index}_{C \ell_{d, 0}}\left(\mathcal{D}^{E}\right):=\left[\operatorname{ker} \mathcal{D}^{E}, \gamma\right] \in \hat{\mathfrak{M}}_{d} / i\left(\hat{\mathfrak{M}}_{d+1}\right) \cong \mathrm{KO}_{d}
$$

Here $\hat{\mathfrak{M}}_{d}$ denotes the set of pairs $(W, h)$, where $W$ is a finite dimensional representation of $C \ell_{d, 0}$ and $h \in \operatorname{End}(W)$ is a $\mathbb{Z}_{2}$-grading on $W$ anticommuting with Clifford generators of $C \ell_{d, 0}$, and let $i: \hat{\mathfrak{M}}_{d+1} \rightarrow \hat{\mathfrak{M}}_{d}$ denotes the map forgetting the action of $d+1$-th Clifford generator. Note that the real $\mathrm{K}$-group $\mathrm{KO}_{d}$ is isomorphic to one of $\mathbb{Z}, \mathbb{Z}_{2}$ or 0 .

On the other hand, the quasi-representation $\pi_{a, E}$ obtained by the GromovLawson construction for $E$ is also real, i.e., each $\pi_{a, E}\left(t_{j}\right)$ is a real orthogonal matrix. In the same way as (1.1), we define the hermitian $C \ell_{d, 0}$-Wilson-Dirac operator $\mathcal{D}_{\mathcal{W}}^{a, E}$ as

$$
\mathcal{D}_{\mathcal{W}}^{a, E}=\mathcal{D}^{a, E}+\gamma \mathcal{W}^{a, E} \in \mathbb{B}\left(\mathcal{H}_{a, E}\right)
$$

where $\mathcal{H}_{a, E}:=\bigoplus_{x \in a \Pi / \Pi} E_{x} \hat{\otimes} \mathcal{S}$. Then the massive hermitian $C \ell_{d, 0}$-WilsonDirac operator $\mathcal{D}_{\mathcal{W}}^{a, E}+m \gamma$ is an invertible self-adjoint operator anticommuting with Clifford generators $e_{j}$. Hence it determines an element

$$
\mathbf{I}_{C \ell_{d, 0}}\left(\mathcal{D}_{\mathcal{W}}^{a, E}+m \gamma\right):=\left[\mathcal{H}_{a, E}, h_{a, E}\right]-\left[\mathcal{H}_{a, E}, \gamma\right] \in \hat{\mathfrak{M}}_{d} / i_{*} \hat{\mathfrak{M}}_{d+1},
$$

where $h_{a, E}=\left(\mathcal{D}_{\mathcal{W}}^{a, E}+m \gamma\right) /\left|\mathcal{D}_{\mathcal{W}}^{a, E}+m \gamma\right|$.

We will extend the argument given in Section 3 and 4 to this setting, we introduce a generalization of Karoubi's definition of the real K-group to Real $\mathrm{C}^{*}$-algebras (cf. [Kub16b, Corollary 5.15]). Here we say that a Real $\mathrm{C}^{*}$-algebra is a $\mathrm{C}^{*}$-algebra $A$ equipped with an antilinear $*$-isomorphic involution $a \mapsto \bar{a}$. For example, let $X$ be a compact Real space, i.e., $X$ is a compact space equipped with an involution $\tau: X \rightarrow X$. Then $A=C(X)$ with $\bar{f}(x)=\overline{f(\tau(x))}$ is a Real $\mathrm{C}^{*}$-algebra. Let $\Delta_{d}$ denote the direct sum of all $\mathbb{Z}_{2}$-graded irreducible representation of $C \ell_{d, 0}$ with the $\mathbb{Z}_{2}$-grading $\gamma$. 
Definition 5.2. Let $A$ be a unital Real $\mathrm{C}^{*}$-algebra. Let us define the set

$\mathcal{F}_{n}^{d}(A):=\left\{s \in A \hat{\otimes} \mathbb{K}\left(\Delta_{d}^{n}\right) \mid s=s^{*}, \bar{s}=s, s^{2}=1, e_{j} s=-s e_{j}\right.$ for $\left.j=1, \cdots, d\right\}$.

We define the Real K-group $\mathrm{KR}_{d}(A)$ as the set of homotopy classes of $\bigcup_{n} \mathcal{F}_{n}^{d}(A)$, where $\mathcal{F}_{n}^{d}(A) \subset \mathcal{F}_{n+1}^{d}(A)$ is defined by $s \mapsto s \oplus \gamma$. The summation is given by the direct sum and the zero element is represented by $\gamma$.

Remark 5.3. We shortly give some remarks on the above definition of the KR-group.

(1) When $d=0, \Delta_{d}$ is the $\mathbb{Z}_{2}$-graded $\mathbb{R}$-vector space $\mathbb{R} \oplus \mathbb{R}^{\mathrm{op}}$. The map $[s] \mapsto[(s+1) / 2]-[(\gamma+1) / 2]$ gives an isomorphism of $\operatorname{KR}_{0}(A)$ and the Grothendieck group of Real (i.e., invariant under the involution) projections in $\bigcup_{n} \mathbb{M}_{n}(A)$.

(2) For a Real space $X$, the group $\mathrm{KR}_{d}(C(X))$ is isomorphic to the Real Kgroup $\mathrm{KR}^{-d}(X)$ defined in [Kar08, Exercise III.7.14]. In particular, when $A=\mathbb{R}$, the group $\mathrm{KR}_{d}(\mathbb{R})$ is isomorphic to $\mathrm{KO}_{d}$ by mapping $[s]$ to $\left[\Delta_{d}^{n}, s\right]$.

(3) The Real K-theory extends to the Kasparov theory. In particular, the index pairing with the Real K-homology cycle $[\mathcal{D}] \in \mathrm{KR}^{-d}(C(M))$ induces a map

$$
\langle\cdot,[\mathcal{D}]\rangle_{A}: \mathrm{KR}_{0}(C(M) \otimes A) \rightarrow \mathrm{KR}_{d}(A) .
$$

This index pairing also satisfies Remark 4.1(1), (2) and (3) by assuming $\phi$ and $\mathcal{E}$ to be Real.

If $\mathcal{H}$ is a real Hilbert space, then $\mathbb{K}:=\mathbb{K}\left(\mathcal{H} \otimes_{\mathbb{R}} \mathbb{C}\right)$ is equipped with a canonical Real $C^{*}$-algebra structure. In the same way as Subsection 3.3 we define the Real C*-algebra $\mathcal{Q}:=\prod_{\mathbb{N}} \mathbb{K} / \bigoplus_{\mathbb{N}} \mathbb{K}$. Then $\left(\pi_{a, E}\right)_{N \in \mathbb{N}}$ gives rise to a Real $*$ homomorphism $\pi^{b}: C^{*} \Pi \rightarrow \mathcal{Q}$, where the Real structure on the group $\mathrm{C}^{*}$-algebra $C^{*} \Pi$ determined by $\bar{t}_{j}=t_{j}$. Note that it is checked in the same way as Lemma 3.10 that $\mathrm{KR}_{d}(\mathcal{Q})$ is isomorphic to $\prod \mathrm{KO}_{d} / \bigoplus \mathrm{KO}_{d}$.

The statement analogous to Lemma 3.6 and Remark 4.1 (4) holds for this Real setting. Note that is identified with that on $C(\hat{\Pi})$ induced from the involution $\tau(\chi)=-\chi$. We write $\mathbb{R}^{p, q}$ for the Real space $\mathbb{R}^{p} \oplus i \mathbb{R}^{q}, \mathbb{D}^{p, q}$ for the unit disk of $\mathbb{R}^{p, q}$ and $S^{p, q}$ for the unit sphere of $\mathbb{R}^{p, q}$.

Lemma 5.4. The following hold.

(1) The function $h:=\sum f_{j} \cdot i x_{j}+\gamma x_{0}: S^{1, d} \rightarrow \operatorname{End}(\mathcal{S})$ is self-adjoint, invertible, Real and anticommutes with the Clifford generators $e_{j}$. Then $[h]=\beta \in \operatorname{KR}_{d}\left(C\left(S^{1, d}\right)\right)$.

(2) The Mishchenko bundle $\mathcal{L}:=\tilde{M} \times_{\Pi} C^{*} \Pi$ is a Real bundle of finitely generated Hilbert $C^{*} \Pi$-modules on $M$. Moreover, $\langle[\mathcal{L}],[\mathcal{D}]\rangle_{C^{*} \Pi}=\beta \in$ $\mathrm{KR}^{d}(\hat{\Pi})$.

Note that Lemma 5.4 (1) is consistent with Lemma 3.6 because $[h]$ in Lemma 5.4 corresponds to the element written as $[h]-[\gamma]$ in Lemma 3.6 through the isomorphism in Remark 5.3(1).

Proof. The proof of Lemma3.6 shows that the map $\mathrm{KR}_{d}\left(C\left(S^{1, d}\right)\right) \rightarrow \mathrm{K}_{d}\left(C\left(S^{d}\right)\right)$ forgetting the Real involution on $C\left(S^{1, d}\right)$ sends $[h]$ to the complex Bott generator $\beta \in \mathrm{K}_{d}\left(C\left(S^{d}\right)\right)$. This shows (1) since the above forgetful map in this degree is an isomorphism. 
Next we show (2). As is stated in [MT16, Section 6], particularly the equation (6.8), the index pairing $\langle[\mathcal{L}], \cdot\rangle_{C^{*} \Pi}$ respects the stable splittings $\mathrm{KO}_{d}(M) \cong$

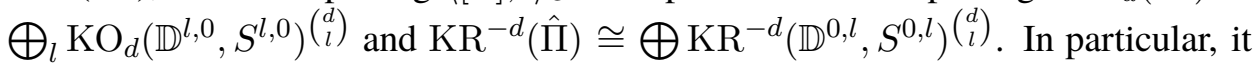
sends $\left[\mathcal{D}\right.$ ] lying in the top degree summand of $\mathrm{KO}_{d}(M)$ to the top degree summand of $\mathrm{KR}^{-d}(\hat{\Pi})$. This shows that $\langle[\mathcal{L}],[\mathcal{D}]\rangle_{C^{*} \Pi}=k \beta$ for some $k \in \mathbb{Z}$. To see that $k=1$, consider the image of $\langle[\mathcal{L}],[\mathcal{D}]\rangle_{C^{*} \Pi}$ to the complex K-group by the forgetful map and remind Remark 4.1 (4).

Remark 5.3 and Lemma 5.4 are enough to check that the same argument as in Sections 3 and 4 also work in this setting. Finally we obtain the following theorem.

Theorem 5.5. The following hold.

(1) For any $0<m<2$, there is $a_{0}=1 / N_{0}$ such that $\mathcal{D}_{\mathcal{W}}^{a, E}+\frac{m}{a}$ is invertible and $\mathbf{I}_{C \ell_{d, 0}}\left(\mathcal{D}_{\mathcal{W}}^{a, E}+\frac{m}{a} \gamma\right)=\operatorname{Index}_{C \ell_{d, 0}}\left(\mathcal{D}^{E}\right)$ holds for any $0<a<a_{0}$.

(2) There is $m_{0}>0$ such that, for any $m>m_{0}$ there is $a_{0}=1 / N_{0}$ such that $\mathcal{D}_{\mathcal{W}}^{a, E}+m \gamma$ is invertible and $\mathbf{I}_{C \ell_{d, 0}}\left(\mathcal{D}_{\mathcal{W}}^{a, E}+m \gamma\right)=\operatorname{Index}_{C \ell_{d, 0}}\left(\mathcal{D}^{E}\right)$ holds for any $0<a<a_{0}$.

Remark 5.6. The index pairing with a quaternionic vector bundle, instead of a real vector bundle, is also treated in a similar way. In this case, $\operatorname{Index}_{C \ell_{d, 0}}\left(\mathcal{D}^{E}\right)$ and $\mathbf{I}_{C \ell_{d, 0}}\left(\mathcal{D}_{\mathcal{W}}^{a, E}+m \gamma\right)$ take value in the group of quaternionic representations of the Clifford algebra $C \ell_{d, 0}$, which is isomorphic to $\mathrm{KO}_{d+4}$.

Indeed, let us identify the quaternion field $\mathbb{H}$ with its complexification $\mathbb{H} \otimes_{\mathbb{R}} \mathbb{C}$ as the Real $C^{*}$-algebra. It is isomorphic to $\mathbb{M}_{2}(\mathbb{C})$ equipped with the Real involution $a \mapsto u \bar{a} u^{*}$, where $\bar{a}$ is the usual complex conjugation and $u=\left(\begin{array}{ll}0 & 1 \\ 1 & 0\end{array}\right)$. Then a quaternionic vector bundle $E$ determines an element of $\mathrm{KR}_{0}(C(M) \otimes \mathbb{H})$ and the sequence $\left(\pi_{a, E}\right)_{N \in \mathbb{N}}$ gives rise to a Real $*$-homomorphism $\pi^{b}: C^{*} \Pi \rightarrow \mathcal{Q} \otimes \mathbb{H}$. Hence the same proofs of Theorem 3.14 and Lemma 4.2 work only by replacing $\mathcal{Q}$ with $\mathcal{Q} \otimes \mathbb{H}$.

\section{REFERENCES}

[Ada02a] David H. Adams, Axial anomaly and topological charge in lattice gauge theory with overlap Dirac operator, Annals of Physics 296 (2002), no. 2, 131-151.

[Ada02b] _ Families index theory for overlap lattice Dirac operator. I, Nuclear Physics. B. Theoretical, Phenomenological, and Experimental High Energy Physics. Quantum Field Theory and Statistical Systems 624 (2002), no. 3, 469-484.

[AS69] Michael Atiyah and I. M. Singer, Index theory for skew-adjoint Fredholm operators, Institut des Hautes Études Scientifiques. Publications Mathématiques 37 (1969), 5-26.

[CD13] José R. Carrión and Marius Dadarlat, Quasi-representations of surface groups, Journal of the London Mathematical Society. Second Series 88 (2013), no. 2, 501-522.

[CGM90] Alain Connes, Mikhail Gromov, and Henri Moscovici, Conjecture de Novikov et fibrés presque plats, Comptes Rendus de l'Académie des Sciences. Série I. Mathématique 310 (1990), no. 5, 273-277.

[Dad12] Marius Dadarlat, Group quasi-representations and index theory, Journal of Topology and Analysis 4 (2012), no. 3, 297-319.

[Dad14] _ Group quasi-representations and almost flat bundles, Journal of Noncommutative Geometry 8 (2014), no. 1, 163-178.

[EL91] Ruy Exel and Terry A. Loring, Invariants of almost commuting unitaries, Journal of Functional Analysis 95 (1991), no. 2, 364-376.

$\left[\mathrm{FFM}^{+}\right]$Hidenori Fukaya, Mikio Furuta, Shinichiroh Matsuo, Tetsuya Onogi, Satoshi Yamaguchi, and Mayuko Yamashita, On analytic indices in lattice gauge theory and their continuum limits, in preparation. 
$\left[\mathrm{FKM}^{+}\right.$19] Hidenori Fukaya, Naoki Kawai, Yoshiyuki Matsuki, Makito Mori, Katsumasa Nakayama, Tetsuya Onogi, and Satoshi Yamaguchi, A lattice formulation of the Atiyah-Patodi-Singer index, PoS Proceedings, 37th International Symposium on Lattice Field Theory (Lattice 2019) (2019), no. OU-HET-1038, 149.

$\left[\mathrm{FKM}^{+} 20\right] \ldots$, The Atiyah-Patodi-Singer index on a lattice, Progress of Theoretical and Experimental Physics 2020 (April 2020), no. 4.

[Fuj99] Kazuo Fujikawa, A continuum limit of the chiral Jacobian in lattice gauge theory, Nuclear Physics B 546 (1999), no. 1, 480-494.

[GL83] Mikhael Gromov and H. Blaine Lawson Jr., Positive scalar curvature and the Dirac operator on complete Riemannian manifolds, Institut des Hautes Études Scientifiques. Publications Mathématiques 58 (1983), 83-196 (1984).

[Gro96] M. Gromov, Positive curvature, macroscopic dimension, spectral gaps and higher signatures, Functional analysis on the eve of the 21st century, Vol. II (New Brunswick, NJ, 1993), 1996, pp. 1-213, Birkhäuser Boston, Boston, MA.

[Hal76] P. R. Halmos, Some unsolved problems of unknown depth about operators on Hilbert space, Proceedings of the Royal Society of Edinburgh: Section A Mathematics 76 (1976), no. $1,67-76$.

[HLN98] Peter Hasenfratz, Victor Laliena, and Ferenc Niedermayer, The index theorem in QCD with a finite cut-off1Work supported in part by schweizerischer nationalfonds, by iberdrola, ciencia y tecnologia, españa and by the ministerio de educacion y cultura, España.1, Physics Letters B 427 (1998), no. 1, 125-131.

[HR00] Nigel Higson and John Roe. Analytic K-homology, Oxford Mathematical Monographs, Oxford University Press, Oxford, ISBN 0-19-851176-0, (2000).

[HS06] B. Hanke and T. Schick, Enlargeability and index theory, Journal of Differential Geometry 74 (2006), no. 2, 293-320.

[Kar08] Max Karoubi. K-theory, Classics in Mathematics, Springer-Verlag, Berlin, ISBN 9783-540-79889-7, (2008).

[Kub16a] Yosuke Kubota, The joint spectral flow and localization of the indices of elliptic operators, Annals of K-Theory 1 (2016), no. 1, 43-83.

[Kub16b] Notes on twisted equivariant $K$-theory for $C^{*}$-algebras, International Journal of Mathematics 27 (2016), no. 6, 1650058, 28.

[Kub20] _ Almost flat relative vector bundles and the almost monodromy correspondence, Journal of Topology and Analysis (2020).

[Lus72] Gheorghe Lusztig, Novikov's higher signature and families of elliptic operators, Journal of Differential Geometry 7 (1972), no. 1-2, 229-256.

[Lüs99] Martin Lüscher, Topology and the axial anomaly in abelian lattice gauge theories, Nuclear Physics B 538 (1999), no. 1, 515-529.

[MT16] Varghese Mathai and Guo Chuan Thiang, T-Duality Simplifies Bulk-Boundary Correspondence: Some Higher Dimensional Cases, Annales Henri Poincaré 17 (January 2016), no. 12, 3399-3424.

[Neu00] Herbert Neuberger, Bounds on the wilson dirac operator, Physical Review D 61 (March 2000), no. 8, 085015.

[NN95] Rajamani Narayanan and Herbert Neuberger, A construction of lattice chiral gauge theories, Nuclear Physics B 443 (1995), no. 1, 305-385.

[PS16] Emil Prodan and Hermann Schulz-Baldes. Bulk and boundary invariants for complex topological insulators, Mathematical Physics Studies, Springer, [Cham], ISBN 978-3319-29350-9 978-3-319-29351-6, (2016).

[RLL00] M. Rørdam, F. Larsen, and N. Laustsen. An introduction to $K$-theory for $C^{*}$-algebras, London Mathematical Society Student Texts, vol. 49, Cambridge University Press, Cambridge, ISBN 0-521-78334-8 0-521-78944-3, (2000).

[Sch05] Thomas Schick, $L^{2}$-index theorems, KK-theory, and connections, New York Journal of Mathematics 11 (2005), 387-443 (electronic).

[Seg77] Graeme Segal, K-homology theory and algebraic K-theory, K-theory and operator algebras (Proc. Conf., Univ. Georgia, Athens, Ga., 1975), 1977, pp. 113-127. Lecture Notes in Math., Vol. 575, Springer, Berlin.

[Suz99] Hiroshi Suzuki, Simple evaluation of chiral Jacobian with overlap Dirac operator, Prog. Theor. Phys. 102 (1999), 141-147. 
[Voi83] Dan Voiculescu, Asymptotically commuting finite rank unitary operators without commuting approximants, Acta Universitatis Szegediensis. Acta Scientiarum Mathematicarum 45 (1983), no. 1-4, 429-431.

[Yam20] Mayuko Yamashita, A lattice version of the Atiyah-Singer index theorem, preprint (2020). arXiv:2007.06239 [math.DG] 